\title{
A complexidade da determinação da obesidade infantil
}

\author{
The complexity of determining childhood obesity
}

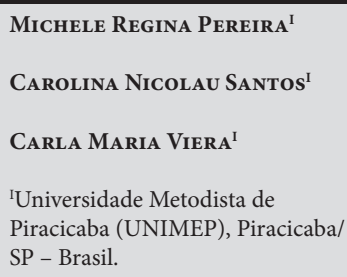

Resumo Segundo a Organização Mundial de Saúde (OMS), nos últimos anos, a obesidade infantil atingiu crianças de todas as faixas etárias e de países desenvolvidos e países em desenvolvimento. Ao menos 41 milhões de crianças de todo o mundo, menores de 5 anos de idade, apresentam sobrepeso/obesidade, de acordo com a OMS. A obesidade é uma doença multifatorial que envolve a interação de vários fatores como biológicos, ambientais e psicossociais, que dificultam a distinção de sua etiologia. A dificuldade na determinação da obesidade infantil sugere, portanto, a necessidade de aprofundamento teórico e identificação da produção científica atual sobre o tema. Diante desse contexto, este estudo realizou uma revisão integrativa, por meio de obras científicas publicadas no período de 2005 a 2017. A busca foi feita nas bases de dados Scielo, Medline e Lilacs. A partir de 14 artigos selecionados e analisados, foi concluído que determinados fatores podem contribuir com a obesidade infantil, sendo eles de contexto familiar, mídia, sedentarismo, urbanização e poder aquisitivo. A associação de dois ou mais determinantes, na obesidade infantil, alerta que, se não controlada, os números de crianças com obesidade podem ser maiores.

Palavras-chave: Obesidade infantil. Origem. Comportamento ALIMENTAR, MUNDIAL E SAÚde PÚbliCa.

Abstract According to the World Health Organization (WHO), in recent years, childhood obesity has affected children of all ages in developed and developing countries. At least 41 million children worldwide, under 5 years old, are overweight/obese. Obesity is a multifactorial disease that involves the interaction of several factors such as biological, environmental and psychosocial that make it difficult to distinguish its etiology. The difficulty in determining childhood obesity suggests, therefore, the need for a theoretical deepening and also the identification of current scientific production on the subject. Due to this context, the present study was carried out from a bibliographical review, through works published in the period from 2005 to 2017. The search was performed in the Scielo, Medline and Lilacs databases. From 14 selected and analyzed articles, it was concluded that indeterminate factors can contribute to childhood obesity, among them the family context, media, sedentarism, urbanization and purchasing power. The association of two or more determinants in childhood obesity warns that if uncontrolled, numbers of children with obesity may be higher.

Key-words: Childhood obesity. ORIGIN. Food behavior, global and Public health. 


\section{INTRODUÇÃO}

A obesidade infantil vem crescendo de forma preocupante. Segundo a Organização Mundial de Saúde (OMS), nos últimos anos a obesidade infantil atingiu crianças de todas as faixas etárias, de países desenvolvidos e países em desenvolvimento. Ao menos 41 milhões de crianças menores de 5 anos de idade já apresentam sobrepeso ou obesidade, sendo que os maiores aumentos desses casos são de países de média e baixa renda. ${ }^{1}$

Há muitos fatores que ajudam no crescimento dos índices de obesidade infantil, e que não apareciam há pouco tempo. Eles vêm sendo observados há cerca de 20 anos. Consta que, em países em desenvolvimento e desenvolvidos, a genética, a falta de exercícios físicos e problemas socioeconômicos, contribuem para o aumento da obesidade infantil, por conseguinte, trazendo as doenças crônicas não transmissíveis. ${ }^{2}$

A atenção deve ser redobrada com o sobrepeso ou a obesidade, na infância, pois o excesso de peso constitui um marcador de risco para a manifestação precoce das doenças crônicas na vida adulta. ${ }^{3}$ A obesidade se constitui de um distúrbio crônico multifatorial, que tem relação com outras doenças crônicas, que debilitam a qualidade de vida. ${ }^{4}$

Doenças crônicas não transmissíveis são consequências de um índice de massa corporal (IMC) elevado para sobrepeso ou obesidade. Fazem parte desse conjunto as doenças cardiovasculares que acompanham a síndrome plurimetabólica. Esta, por sua vez, é um transtorno complexo representado por um conjunto de riscos associados ao excesso de gordura corporal, como hipertensão, diabetes mellitus e dislipidemia que, se não tratados, podem estender-se na fase adulta. O sedentarismo e uma dieta inadequada têm associações significativas para o excesso de peso., ${ }^{5,6,3}$

A obesidade é mais que um problema individual. É um problema social, que traz fatores negativos na infância. Alguns estudos relatam que as crianças sofrem bullying e são chamadas de burras e preguiçosas. ${ }^{4}$

A obesidade traz implicações na imagem corporal das crianças e no desenvolvimento físico. Atividades físicas trazem benefícios para melhor controle de peso, provocam o bem-estar, proporcionando mais disposição no desenvolvimento da vida. No entanto, encontram-se barreiras que impedem a disposição para atividades físicas na infância, com distrações do lazer passivo, de ficar à frente da televisão e com jogos de game, desmotivando o lazer ativo, que era mais comum como pular corda, andar de bicicleta e patins. ${ }^{7,89}$

A dificuldade na determinação da obesidade infantil sugere, portanto, a necessidade de aprofundamento teórico e identificação da produção científica atual sobre o tema. $\mathrm{O}$ objetivo desta revisão bibliográfica é identificar fatores que contribuem para o aumento da obesidade infantil.

\section{Metodologia}

Trata-se de um estudo de revisão integrativa que é um método que proporciona uma integração de opiniões e conceitos, do qual se tem uma coleta de dados realizada por intermédio de fontes secundárias por meio de levantamento bibliográfico. ${ }^{10}$

O presente trabalho tem como base de revisão integrativa a proposta pelo Manual 
de revisão bibliográfica sistemática integrativa, ${ }^{11}$ que consiste de seis etapas:

$1^{\circ}$. identificação do tema para o trabalho, com a formulação de uma pergunta norteadora e a definição de descritores.

$2^{\circ}$. critérios para inclusão e exclusão de dados e o início da busca de estudos.

$3^{\circ}$. amostragem/coleta de dados por meio de palavras-chave, por título de artigos e por leitura dos resumos dos artigos.

$4^{\circ}$. análise crítica dos estudos inclusos e leitura dos estudos completos.

$5^{\circ}$. análise e discussão de resultados.

$6^{\circ}$. apresentação da revisão, proposta para estudos futuros.

Os artigos foram selecionados utilizando as bases de dados Scientific Electronic Library Online (Scielo), Medical Literature Analysis and Retrieval System Online (Medline) e Literatura Latino-Americana e do Caribe em Ciências da Saúde (Lilacs), por meio dos seguintes descritores validados nos Descritores em ciências da saúde (Decs): ${ }^{11}$ Obesidade infantil, origem, comportamento alimentar, mundial e saúde pública.

Para guiar este estudo, foi elaborada a seguinte questão "Quais os fatores determinantes para o aumento da obesidade infantill?", tendo como critério de inclusão artigos originais publicados em português, inglês e espanhol entre os anos de 2005 a 2017 e disponíveis na íntegra, e como critérios de exclusão foram excluídos artigos de revisão bibliográfica, teses, livros e de acesso restrito.

A partir dos descritores, foram encontrados 53 artigos. Foi realizada a leitura do resumo dos artigos e foram excluídos 27 artigos. Após a leitura na íntegra, foram selecionados 14 artigos para a elaboração do presente estudo (Quadro 1).

\section{Obesidade infantil e o contexto familiar}

Em estudo realizado por Melzer et al., ${ }^{12}$ entre as mães que apresentam gordura abdominal, existe uma chance de 2,7 vezes maior que seus filhos tenham o mesmo quadro. Foi constatado, também, que a obesidade infantil com um índice de 30,7\% se relaciona com a ocorrência de excesso de peso materno com um índice de 64\%, especialmente para as crianças do sexo feminino.

Fernandes et al. ${ }^{13}$ identificaram que a obesidade não só no sexo feminino, também no sexo masculino é um reflexo da obesidade da mãe e do pai. Esse mesmo estudo mostra que a obesidade na adolescência não só está associada com a gordura abdominal da mãe, como cita Melzer et al., ${ }^{12}$ ela também está associada com a gordura abdominal da figura paterna.

No estudo de Schuch et al., ${ }^{14}$ famílias que apresentam a figura paterna em seus lares demonstraram a predominância de $12 \%$, correspondente a 436 famílias com excesso de peso infantil. Estudos complementares são necessários para conhecer melhor as causas dessa determinação da obesidade infantil, ou seja, compreender melhor por que a presença paterna, em determinados estudos, vincula-se à obesidade infantil.

Não só a presença dos pais pode influenciar na obesidade infantil, mas também os números de integrantes na mesma residência podem induzir na maior ou menor prevalência da obesidade infantil. Como observado no estudo de Schuch et al., ${ }^{14} \mathrm{o}$ número de pessoas que vivem no domicílio é um possível determinante da obesidade infantil. Famílias maiores ou iguais a seis integrantes apresentam 6,5\% e famílias com três ou menos integrantes apresentam $14,7 \%$ em obesidade infantil. ${ }^{14}$ 


\section{Quadro 1: Síntese dos artigos analisados}

\begin{tabular}{|c|c|c|}
\hline$N^{0}$. referência & População & Objetivo \\
\hline 12 & $\begin{array}{l}357 \text { pares de mães e crianças de } 3 \\
\text { a } 10 \text { anos de idade. }\end{array}$ & $\begin{array}{l}\text { Analisar a associação de fatores dietéticos, socioeconômicos, comportamentos } \\
\text { sedentários e do estado nutricional materno com acúmulo de gordura abdominal de } \\
\text { crianças de } 3 \text { a } 10 \text { anos de idade. }\end{array}$ \\
\hline 13 & $\begin{array}{l}691 \text { adolescentes de Presidente } \\
\text { Prudente (SP). }\end{array}$ & $\begin{array}{l}\text { Analisar a associação entre fatores de risco familiares e a presença de obesidade } \\
\text { abdominal entre adolescentes. }\end{array}$ \\
\hline 14 & $\begin{array}{l}4.914 \text { crianças de escola pública } \\
\text { do Estado do (RS) e (SC). }\end{array}$ & $\begin{array}{l}\text { Determinar a prevalência de excesso de peso e os seus fatores associados em crianças } \\
\text { pré-escolares. }\end{array}$ \\
\hline 16 & Oito famílias soteropolitanas. & Aprofundar a compreensão da dinâmica familiar de filhos únicos obesos na infância. \\
\hline 17 & $\begin{array}{l}793 \text { crianças menores de } 4 \text { anos de } \\
\text { idade. }\end{array}$ & $\begin{array}{l}\text { Descrever a prevalência e os fatores determinantes do sobrepeso de crianças menores } \\
\text { de } 4 \text { anos de idade de Feira de Santana (BA). }\end{array}$ \\
\hline 18 & $\begin{array}{l}1.435 \text { crianças e adolescentes de } 5 \\
\text { a } 19 \text { anos de idade de } \\
\text { Pernambuco. }\end{array}$ & $\begin{array}{l}\text { Verificar a prevalência e os determinantes do excesso ponderal em crianças e } \\
\text { adolescentes. }\end{array}$ \\
\hline 19 & $\begin{array}{l}2.035 \text { adolescentes de escola } \\
\text { pública e privada. }\end{array}$ & $\begin{array}{l}\text { Analisar a prevalência e os fatores sociodemográficos associados à presença isolada e } \\
\text { combinada de indicadores antropométricos elevados em crianças. }\end{array}$ \\
\hline 20 & $\begin{array}{l}57 \text { escolares de } 7 \text { a } 10 \text { anos de } \\
\text { idade do ensino fundamental de } \\
\text { escola particular. }\end{array}$ & $\begin{array}{l}\text { Identificar o comportamento consumidor de escolares de escola particular em relação } \\
\text { aos seus hábitos alimentares e à influência da televisão. }\end{array}$ \\
\hline 21 & $\begin{array}{l}1.158 \text { da rede de ensino público e } \\
\text { privado do município de } \\
\text { Fortaleza. }\end{array}$ & $\begin{array}{l}\text { Determinar a prevalência de sobrepeso e obesidade em escolares e estimar a diferença } \\
\text { entre a prevalência nas escolas públicas e privadas, segundo sexo e faixa etária } \\
\text { (adolescência precoce e tardia). }\end{array}$ \\
\hline 22 & $\begin{array}{l}200 \text { crianças de escola pública e } \\
\text { particular de } 8 \text { a } 10 \text { anos de idade. }\end{array}$ & $\begin{array}{l}\text { Diagnosticar e comparar a prevalência de sobrepeso e obesidade em crianças de duas } \\
\text { escolas da cidade de São Paulo, com características socioeconômicas diferentes. }\end{array}$ \\
\hline 23 & $\begin{array}{l}782 \text { escolares do } 1^{\circ} \text {. ano do ensino } \\
\text { fundamental da rede municipal. }\end{array}$ & $\begin{array}{l}\text { Verificar a associação entre insegurança alimentar medida com a Escala Brasileira de } \\
\text { Insegurança Alimentar (EBIA) e o excesso de peso em crianças. }\end{array}$ \\
\hline 24 & 1.616 crianças e adolescentes. & $\begin{array}{l}\text { Comparar a prevalência de sobrepeso e obesidade em pré-escolares, escolares e } \\
\text { adolescentes (escola pública e privada) de condições socioeconômicas distintas. }\end{array}$ \\
\hline 25 & $\begin{array}{l}354 \text { adolescentes de } 17 \text { a } 19 \text { anos } \\
\text { de idade de escolas públicas do } \\
\text { município. }\end{array}$ & $\begin{array}{l}\text { Verificar associações entre estado antropométrico e sexo, entre os adolescentes } \\
\text { participantes do estudo, para que estratégias de educação alimentar possam ser } \\
\text { sugeridas. }\end{array}$ \\
\hline 26 & $\begin{array}{l}\text { Crianças de } 24 \text { a } 59 \text { meses, pré- } \\
\text { escolares. }\end{array}$ & $\begin{array}{l}\text { Conhecer a frequência de consumo de bebidas açucaradas (BA) entre crianças } \\
\text { brasileiras e investigar associações com variáveis demográficas, socioeconômicas e } \\
\text { nutricionais. }\end{array}$ \\
\hline
\end{tabular}


Para fortalecer essa abordagem, os dados do Instituto Brasileiro de Geografia e Estatística (IBGE) $)^{15}$ mostram que há tendência de queda do número de filhos nas famílias brasileiras, bem como, de outros países, o que leva a compreender que, com a queda do número de pessoas nas famílias, pode agravar a tendência de aumento da obesidade infantil.

Se levarmos em conta a lógica de determinação apontada por Schuch et al., ${ }^{14} \mathrm{e}$ Santos e Rabinovich, ${ }^{16}$ com relação ao filho único, há uma grande chance de apresentar obesidade infantil. Essa afirmação não significa que toda criança filho único terá obesidade infantil, mas que há uma grande chance.

Santos e Rabinovich ${ }^{16}$ fizeram um estudo qualitativo com oito famílias que tinham apenas um filho, e todos os filhos apresentavam obesidade grave. Esse estudo apontou alguns possíveis determinantes para ocorrer a obesidade infantil, como uma gravidez inesperada e até mesmo por instabilidade conjugal. Essas crianças vivem sem o afeto e carinho necessários que seriam dados pelos pais, e então buscam nos alimentos essa falta em sua existência.

...por isso, o filho único pode vir a encontrar, $\operatorname{logo}$ ao nascer, condições propiciadoras a que sua vinculação básica com a figura materna não se processe de modo pleno, ocasionando que parte do que não recebe seja derivada para uma satisfação no alimento. ${ }^{16}$

Uma análise feita no estudo de Jesus et al. ${ }^{17}$ demonstrou que crianças que são filhos primogênitos e únicos apresentam um índice 1,6 vezes maior de sobrepeso infantil. No estudo, é citado que, ao ter mais filhos, torna-se um protetor ao sobrepeso infantil. ${ }^{17}$
A escolaridade da mãe pode ser também um dos determinantes para a obesidade infantil. Foram identificados 16\% de crianças com sobrepeso no estudo de Jesus et al., ${ }^{17}$ em que as mães tinham escolaridade maior. Por consequência, o fato dessas mães terem uma condição socioeconômica melhor apresenta uma possível facilidade em adquirir alimentos industrializados e com alto valor calórico para os seus filhos.

...É importante salientar que mulheres com
maior escolaridade podem apresentar também
poder aquisitivo mais elevado, com maior chan-
ce de adquirir alimentos industrializados e mais
calóricos. ${ }^{17}$

Schuch et al. ${ }^{14}$ relatam diferenças dos resultados realizados por Jesus et al. ${ }^{17} \mathrm{Schu}$ ch et al. ${ }^{14}$ apontam que crianças, filhos de mãe com ensino fundamental ou ensino superior, apresentam menor taxa em excesso de peso, $10,5 \%$ e $9,8 \%$ respectivamente. Nas famílias de menor renda, os alimentos tendem a ser divididos em número maior de parcelas, o que justifica as taxas menores de excesso de peso. Nas famílias de maior renda, com mães de um nível de escolaridade maior, poderá haver um maior conhecimento de alimentação saudável, e condições melhores em incorporar nas refeições melhor qualidade, podendo incluir frutas, verduras e legumes nas refeições diárias. No entanto, os autores não discutem os determinantes da obesidade infantil nas famílias intermediárias.

Em seu estudo, Jesus et al. ${ }^{17}$ demonstram que determinantes da obesidade infantil relacionam-se também com o retorno precoce da mãe ao trabalho. O retorno acontece geralmente quando o seu filho ainda 
está com 4 meses de idade. Foi identificado um aumento de 1,7 vezes a mais de sobrepeso em crianças que a mãe volta ao trabalho mais cedo. Nesses casos, há maior probabilidade do desmame precoce. A criança com a alimentação de adultos pode ser um dos fatores da obesidade infantil

...de que as crianças cujas mães já trabalham no quarto mês após seu nascimento são mais precocemente desmamadas e introduzidas no consumo dos alimentos da família, estando mais susceptíveis ao sobrepeso. ${ }^{17}$

Outro fator que o estudo de Jesus et al. ${ }^{17}$ aponta como sendo um indicativo considerável do sobrepeso infantil são as mães que precisam passar o dia fora de casa, trabalhando para o sustento da família, ausentando-se do lar por longos períodos. Essa constatação associa-se como determinante da obesidade infantil pela compensação com alimentos, como um comportamento comum entre mães ausentes.

...Além disso, mães que trabalham fora do lar têm uma tendência de agradar os filhos com merendas, que na maioria das vezes apresentam alto valor calórico e baixo valor nutricional. ${ }^{17}$

\section{A urbanização: obesidade infan- til na cidade e no meio rural}

Um estudo realizado em Pernambuco por Leal et al. ${ }^{18}$ mostrou que famílias do interior urbano apresentaram uma predominância de $20,7 \%$ de excesso de peso, na região metropolitana $16,2 \%$ e $9,8 \%$ no interior rural.

A relevância desse agravo que atinge $o$ interior urbano mostra que a facilidade ao acesso a alimentos industrializados e redes de fast foods cresceu no interior, ficando semelhante à região metropolitana. ${ }^{18}$

Nesse mesmo estudo, Leal et al. ${ }^{18}$ indicaram que a obesidade no meio rural encontra-se acima da taxa preconizada pela OMS de $2,3 \%$, estando quatro vezes acima da taxa. Nesse trabalho esses números, apesar de não serem tão decisivos quanto no meio urbano, demonstram que a obesidade também está presente de forma significante no meio rural.

Os autores justificam neste estudo ${ }^{18}$ que na região rural esses percentuais estão abaixo devido ao estilo de vida desses moradores e da falta de disponibilidade de espaço para redes de fast foods. Entretanto, a geração de empregos e melhores condições econômicas oferecidas às famílias dessas regiões contribuem para a entrada de alimentos processados, por conseguinte contribuindo para $o$ aumento da obesidade infantil.

Barbosa Filho et al., ${ }^{19}$ em seu estudo realizado em Colombo (PR), avaliaram a presença de indicadores antropométricos de 2.035 crianças. Foi notado que, na região rural, $22 \%$ das crianças apresentaram excesso de peso e, na região urbana, 9,2\% com excesso de peso. O referido estudo aponta que essas taxas, acima da recomendação da OMS, na região rural, são decorrentes do aumento acentuado no acesso e aquisição de alimentos de alta densidade energética, além da falta de atividade física. Segundo os autores, essas regiões mais afastadas dos centros urbanos são grandes potencializadores para o desenvolvimento de obesidade infantil.

\section{Diferenças regionais}

Em cada região brasileira a obesidade infantil tem se manifestado de forma distinta. Estudos da literatura nos mostram que 
a obesidade infantil pode variar, de acordo com a região/localidade. Na região Sul, Fiates, Amboni e Teixeira ${ }^{20}$ nos mostram que em seu estudo realizado em Florianópolis (SC) a prevalência de excesso de peso em crianças foi de 23,6\%. Nessa mesma região, Schuch et al., ${ }^{14}$ em seu estudo realizado com 4.914 crianças, mostraram que 14,4\% de crianças do Rio Grande do Sul (RS) e 7,5\% de Santa Catarina (SC) apresentaram excesso de peso. Na região Sudeste, Melzer et al., ${ }^{12}$ no estudo realizado em Santos (SP), encontraram uma prevalência de 30,5\% de excesso de peso em crianças. $\mathrm{Na}$ região do Nordeste, Campos, Leite e Almeida, ${ }^{21}$ no estudo realizado em Fortaleza (CE), encontraram uma prevalência de 19,5\% de excesso de peso nas crianças avaliadas.

Essas comparações dos dados apresentados nos estudos deixam evidente que a obesidade infantil encontra-se muito acima do que é aceitável pela OMS, porém com variações regionais importantes. As diferenças regionais de prevalência não esclarecem, contudo, as diferenças na determinação, que obviamente existem, mas é necessário mais pesquisas para a sua elucidação.

Ao conhecer as diferenças de determinação regional, programas de prevenção e controle retomam com maiores possibilidades de implementação. Cada região tem uma característica, diferenciação social e cultural, e esses programas e controles devem estar voltados para as particularidades de cada região.

\section{Diferenças entre escola privada e pública e o poder aquisitivo na determinação da obesidade infantil}

Miranda et al., ${ }^{22}$ em estudo realizado em São Paulo com 200 escolares de rede pública e privada, encontraram uma prevalência de sobrepeso de $19,6 \%$ nos meninos e $25,5 \%$ nas meninas de escola privada e $7 \%$ nos meninos e $8,1 \%$ nas meninas de escola pública.

Campos, Leite e Almeida, ${ }^{21}$ em estudo realizado com 1.158 escolares de escolas privada e pública de Fortaleza, encontraram excesso de peso de $23,9 \%$ em crianças de escola privada e $18 \%$ em escola pública.

Contrariando os achados de Miranda et al., ${ }^{22}$ e de Campos, Leite e Almeida, ${ }^{21} \mathrm{em}$ um estudo realizado com 2.035 escolares do Paraná (PR), Barbosa Filho et al. ${ }^{19}$ encontraram que as crianças matriculadas em rede particular tiveram menor indicadores de excesso de peso quando comparado às crianças de rede pública $(3,1 \%$ na rede particular e $25 \%$ na rede pública).

Estudos recentes indicam que o desenvolvimento para o aumento da obesidade infantil também tem relação com os níveis socioeconômicos da família. Vicenzi et al., ${ }^{23}$ em estudo em São Leopoldo (RS), encontraram uma predominância de $38,1 \%$ de excesso de peso nos escolares estudados, e a maior parte era de menor poder aquisitivo.

Em um estudo realizado em Recife (PE), com 1.616 crianças de diferentes condições econômicas, ${ }^{24}$ encontraram uma predominância de 17,4\%,10,8\% e 4,4\% de obesidade em pré-escolares, escolares e adolescentes de maior poder aquisitivo e 10,1\%, 3\% e 5,6\% de obesidade, respectivamente, nos de menor poder aquisitivo. Segundo os autores, a obesidade nos pré-escolares e escolares de maior poder aquisitivo se mostrou mais prevalente devido à mudança no padrão alimentar e aumento no consumo de açúcares, gorduras e na redução de fibras em sua alimentação. ${ }^{24}$ 
Santos et al., ${ }^{25}$ em estudo realizado por meio de dados antropométricos com 354 adolescentes da Bahia, encontraram uma predominância de $4 \%$ de excesso de peso nesses adolescentes e $91,8 \%$ de Eutrofia. A maior parte desses adolescentes pertencia a famílias de baixo poder aquisitivo.

Segundo os autores, a idade desses adolescentes é próxima à fase de inserção no mercado de trabalho, e esses adolescentes procuram ajudar os pais com as despesas de casa. Em consequência de estarem inseridos no mercado de trabalho, se têm alto gasto energético, nem sempre esse gasto é reposto corretamente, por isso os índices de obesidade são menores nessa idade. ${ }^{25}$

Vega, Poblacion e Taddei, ${ }^{26}$ em estudo realizado com 2.881 pré-escolares, por meio de dados secundários da Pesquisa Nacional de Demografia e Saúde da Criança e da Mulher (PNDS) de 2.006, encontraram que em regiões de melhor desenvolvimento econômico as crianças apresentaram predominância de $43 \%$ de sobrepeso e $49,2 \%$ de obesidade. Crianças de família de maior poder aquisitivo têm maiores risco de obesidade, principalmente por consumirem constantemente em sua alimentação bebidas açucaradas. ${ }^{26}$

Esses estudos nos mostram que a obesidade infantil está presente em escolas públicas e particulares. Essa constatação feita a partir da revisão nos leva a crer que a determinação da obesidade infantil envolve fatores que vão além do poder aquisitivo da família.

\section{A Influência da TV e Mídia}

Em estudo realizado em Florianópolis, ${ }^{20}$ foi observado um índice de $23,6 \%$ de obesidade e sobrepeso infantil. Os autores puderam observar que as crianças passam um longo tempo em frente aos televisores nos momentos em que estão em seus lares, $75 \%$ das crianças assistem mais de quatro vezes ao dia a TV. O referido estudo discute a relação da obesidade infantil com influência da mídia defendendo a ideia, que longos períodos passados em frente aos televisores podem trazer maus comportamentos alimentares e consequências nada favoráveis à saúde. Essas crianças na frente da TV, na maioria das vezes, estão ingerindo alimentos com alto valor calórico e com pouca ingestão de frutas, verduras e legumes, e sem praticar atividades físicas, gerando o sobrepeso e a obesidade infantil. ${ }^{20}$

Em estudo, Leal et al. ${ }^{18}$ observaram que crianças que residem em centros urbanos seus horários de lazer não são mais brincando na rua com os amigos, andando de bicicletas, correndo no quintal. Esses hábitos ficaram para a geração passada. Segundo esses autores, atualmente as crianças passam mais horas na frente da TV assistindo, jogando videogames e no computador, tornando-se crianças sedentárias. Esses hábitos levam as crianças a não praticar exercícios físicos com frequência e isso gera o sobrepeso e a obesidade, o que reforça os resultados apontados em outro estudo. ${ }^{18,20}$

Foi observado por Vega, Poblacion e Taddei, ${ }^{26}$ que o consumo de bebidas açucaradas entre crianças que vivem nas regiões brasileiras mais desenvolvidas economicamente, o Sul, apresentou $51 \%$ e o Sudeste $41,4 \%$, nas classes socioeconômicas mais abastadas. Nesse mesmo estudo, crianças, cujas mães têm por hábito assistir TV com frequência, apresentaram 36,7\% maior risco de consumi- 
rem bebidas açucaradas, e por conseguinte, são crianças com excesso de peso.

\section{Considerações Finais}

Este trabalho destaca os possíveis determinantes para a obesidade infantil no Brasil. Mediante o exposto, conclui-se que os determinantes da obesidade infantil estão relacionados a diversos fatores. No contexto familiar, foi identificado pelos autores revisados que a obesidade infantil se vincula à gordura abdominal da mãe, à presença da figura paterna e materna nas famílias, à quantidade de moradores da residência, ao grau de escolaridade das mães, ao desmame precoce, e ao tempo de retorno das mães, no período pós-parto, ao trabalho.

Outro fator encontrado neste trabalho como determinante da obesidade infantil foi a urbanização. A obesidade infantil encontra-se presente em todas as regiões. Nos estudos revisados, foi possível identificar que alimentos ultraprocessados chegam a todas as regiões, independente da distância e, estes, aliado à falta de exercício físico nas horas de lazer, contribuem para o aumento da obesidade infantil.

O poder aquisitivo faz mudanças no comportamento alimentar e nos padrões de vida dentro e fora das escolas. Foi possível identificar que o poder aquisitivo faz parte do determinante da obesidade infantil, podendo ser maior em famílias de condições socioeconômicas mais elevada como também nas mais baixas. Contudo, a obesidade infantil envolve fatores que vão além do poder aquisitivo.

Outro fator que pode ser considerado um determinante da obesidade infantil são a TV e a Mídia, tendo influência nos hábitos alimentares. A publicidade de alimentos com alto valor calórico faz que as crianças peçam aos pais esses alimentos. Essas crianças passam longo tempo em frente aos televisores e computadores ingerindo alimentos mais calóricos, sem ter a real consciência da quantidade e da qualidade do que estão ingerindo.

A hipótese de o comportamento materno ser o fator determinante para o aumento da obesidade infantil foi superada na medida em que a análise dos artigos revisados proporcionou uma visão muito mais ampla sobre o problema. Muitos determinantes da obesidade infantil foram identificados, o que contribui para qualificar a atuação dos profissionais da saúde, em geral, e especificamente dos nutricionistas, no enfrentamento desse difícil e complexo problema de saúde pública, assim como, no cuidado nutricional dos indivíduos/famílias que buscam assistência.

\section{REFERÊNCIAS}

1. Organização Mundial da Saúde - OMS. Relatório da comissão pelo fim da obesidade infantil busca reverter aumento de sobrepeso e obesidade [on-line] 2016 [acesso em 17 de abril de 2017]. Disponível em http://www.paho.org/bra/index.php?option=com_content\&view=article \&id=4997:relatorio-da-comissao-pelo-fim-da-obesidade-infantil-busca-reverter-aumento-de-sobrepeso-e-obesidade\&Itemid=821.

2. Kreichauf, S., Wildgruber, A., Krombholz, H., Gibson, EL, Vögele, C., Nixon, CA. et al. Revisão narrativa crítica para identificar estratégias educacionais que promovam a atividade física na 
pré-escola [ONLINE] Obes. Rev. 2012, 13: 96-105.

3. Moraes LI, Nicola TC, Jesus JSA, Alves ERB, Giovaninni NPB, Marcato DG. et al. Pressão arterial elevada em crianças e sua correlação com três definições de obesidade infantil. Arq. Bras. Cardiol. [Internet]. 2014; 102 (2): 175-180.

4. Moraes AL, Almeida EC, Souza LB. Percepções de obesos deprimidos sobre os fatores envolvidos na manutenção da sua obesidade: investigação numa unidade do Programa Saúde da Família no município do Rio de Janeiro. Physis [Internet]. 2013; 23 (2): 553-572.

5. Organização Mundial da Saúde - OMS. Obesidade e excesso de peso [on-line] 2016. [Acesso em 17 de abril de 2017]. Disponível em: http://www.who.int/mediacentre/factsheets/fs311/es/.

6. I Diretriz Brasileira de Diagnóstico e Tratamento da Síndrome Metabólica. Arq. Bras. Cardiol. [Internet]. 2005; 84 (Suppl 1): 3-28.

7. Fermino RC, Rech CR, Hino AAF, Rodriguez ACR, Reis RS. Atividade física e fatores associados em adolescentes do ensino médio de Curitiba, Brasil. Rev. Saúde Pública [Internet]. 2010; 44 (6): 986-995.

8. Santos MS, Hino AAF, Reis RS, Rodriguez-Añez CR. Prevalência de barreiras para a prática de atividade física em adolescentes. Rev. bras. Epidemiol. [Internet]. 2010; 13 (1): 94-104.

9. Rodríguez RYL, Argüello GYP. Programas de promoción y prevención para el abordaje de la obesidade infantil: una revisión sistemática. Hacia promoc. Salud [Internet]. 2014; 19 (2): 111126.

10. Cunha, PLP. Manual Revisão Sistemática Integrativa: a pesquisa baseada em evidências. Belo Horizonte: Grupo Ănima Educação, 2014. [Acesso em 12 de maio de 2017]. Disponível em: http://disciplinas.nucleoead.com.br/pdf/anima_tcc/gerais/manuais/manual_revisao.pdf

11. Biblioteca Virtual em Saúde (BIREME). Descritores em Ciências da Saúde- DEC'S [Internet]. [Acesso em 20 de novembro de 2015]. Disponível em: http://decs.bvs.br

12. Melzer MRTF, Magrini IM, Domene SMÁ, Martins PA. Fatores associados à obesidade abdominal em crianças. Rev. paul. Pediatr. [Internet]. 2015; 33 (4): 437-444.

13. Fernandes RA, CJ, Christofaro DGD, Cucato GG, Oliveira AR, Freitas JIF. Fatores familiares associados à obesidade abdominal entre adolescentes. Rev. Bras. Saúde Mater. Infantil [Internet]. 2009; 9 (4): 451-457.

14. Schuch I., Castro TG, Vasconcelos FAG, Dutra CLC, Goldani MZ. Excesso de peso em crianças de pré-escolas: prevalência e fatores associados. J. Pediatr. (Rio J.) [Internet]. 2013; 89 (2): 179188.

15. Instituto Brasileiro de Geografia e Estatística - IBGE. A Síntese dos Indicadores Sociais - Uma Análise das Condições de Vida da População Brasileira. 2008 [Acesso em: 4 de maio de 2017]. Disponível em: http://www.ibge.gov.br

16. Santos LRC, Rabinovich EP. Situações familiares na obesidade exógena do filho único. Saúde soc. [Internet]. 2011; 20 (2): 507-521.

17. Jesus GM, Vieira GO, Vieira TO, Martins CC, Mendes CMC, Castelão ES. Fatores determinantes do sobrepeso em crianças menores de 4 anos de idade. J. Pediatr. (Rio J.) [Internet]. 2010; 86 (4): 311-316.

18. Leal VS, Lira PIC, Oliveira JS, Menezes RCE, Sequeira LAS, Arruda NMA et al. Excesso de peso em crianças e adolescentes no Estado de Pernambuco, Brasil: prevalência e determinantes. Cad. Saúde Pública [Internet]. 2012; 28 (6): 1.175-1.182.

19. Barbosa Filho VC, Campos W, Fagundes RR, Lopes AS, Souza EA. Presença isolada e combinada de indicadores antropométricos elevados em crianças: prevalência e fatores sociodemográficos associados. Ciênc. Saúde coletiva [Internet]. 2016; 21 (1): 213-224. 
20. Fiates GMR, Amboni RDMC, Teixeira E. Comportamento consumidor, hábitos alimentares e consumo de televisão por escolares de Florianópolis. Rev. Nutr. [Internet]. 2008; 21 (1): 105114.

21. Campos LA, Leite ÁJM, Almeida PC. Prevalência de sobrepeso e obesidade em adolescentes escolares do município de Fortaleza, Brasil. Rev. Bras. Saúde Mater. Infant. [Internet]. 2007; 7 (2): 183-190.

22. Miranda JMQ, Palmeira MV, Polito LFT, Brandão MRF, Bocalini DS, Figueira JAJ. et al. Prevalência de sobrepeso e obesidade infantil em instituições de ensino: públicas vs. privadas. Rev Bras Med Esporte [Internet]. 2015; 21 (2): 104-107.

23. Vicenzi K., Henn RL, Weber AP, Backes V., Paniz VMV, Donatti T. et al. Insegurança alimentar e excesso de peso em escolares do primeiro ano do Ensino Fundamental da rede municipal de São Leopoldo, Rio Grande do Sul, Brasil. Cad. Saúde Pública [Internet]. 2015; 31 (5): 1.084-1.094.

24. Silva GAP, Balaban G., Motta MEFA. Prevalência de sobrepeso e obesidade em crianças e adolescentes de diferentes condições socioeconômicas. Rev. Bras. Saúde Mater. Infant. [Internet]. 2005; 5 (1): 53-59.

25. Santos JS, Costa MCO, Nascimento SCL, Silva MCM, Souza KEP, Melo BO. Perfil antropométrico e consumo alimentar de adolescentes de Teixeira de Freitas - Bahia. Rev. Nutr. [Internet]. 2005; 18 (5): 623-632.

26. Vega JB, Poblacion AP, Taddei José AAC. Fatores associados ao consumo de bebidas açucaradas entre pré-escolares brasileiros: inquérito nacional de 2006. Ciênc. Saúde coletiva [Internet]. 2015; 20 (8): 2.371-2.380.

\section{DADOS DAS AUTORAS}

\section{MICHELE REGINA PEREIRA}

Graduanda em Nutrição na Universidade Metodista de Piracicaba. Piracicaba/SP - Brasil. michelllepereira@outlook.com

\section{CAROLINA NICOLAU SANTOS}

Graduanda em Nutrição na Universidade Metodista de Piracicaba. Piracicaba/SP - Brasil. karolnick@hotmail.com

\section{CARLA MARIA VIERA}

Doutora em Psicologia Médica e Psiquiatria pela Universidade Estadual de Campinas. Docente da Universidade Metodista de Piracicaba. Piracicaba/SP - Brasil. carlamariavieira@ hotmail.com

Submetido em: 2-8-2017

Aceito em: 9-10-2017 